\title{
Journal Article Tag Suite 1.0: National Information Standards Organization standard of journal extensible markup language
}

\author{
Sun Huh \\ Department of Parasitology and Institute of Medical Education, College of Medicine, Hallym University, Chuncheon, Korea
}

Abstract

In the era of information technology, scholarly journals cannot escape the rising tide of technological advancement. To be exposed more easily to readers, the web forms of scholarly journals and articles become more important year after year. Furthermore, there is a trend of print journals closing, and a significant emergence of online journals. Journal Article Tag Suite (JATS) extensible markup language (XML) became an National Information Standards Organization standard language in online journal publishing in 2012. It is an essential format to present readers with a more user-friendly interface. JATS XML was developed by PubMed Central (PMC) XML, which was a deposit form of articles to PMC. Editors and other publishing-related personnel should be able to understand the concept and production process of XML files. When JATS XML is produced, a variety of web presentation views can be generated, such as PubReader and epub 3.0. Further, JATS XML can be easily converted to digital object identifier CrossRef XML, CrossMark XML, and FundRef XML. Small scholarly society journal editors and publishers can promote the visibility of their journals by depositing JATS XML files to PMC or ScienceCentral. Owing to these benefits of JATS XML, publishers and editors should now adopt JATS XML for journal publishing.

Received: June 5, 2014

Accepted: July 29, 2014

Correspondence to Sun Huh shuh@hallym.ac.kr

ORCID

Sun Huh

http://orcid.org/0000-0002-8559-8640

This article was presented at the workshop entitled 'Application of advanced

information technology to scholarly journal publishing' during the 12th EASE General Assembly and Conference, Split, Croatia July 12-13, 2014.
Keywords

Extensible markup language; Information technology; Journal Article Tag Suite; Journal publishing; Open access

\section{Introduction}

Journal Article Tag Suite (JATS) extensible markup language (XML) became a National Information Standards Organization (NISO) standard of the journal XML in 2012. In this article,

This is an open access article distributed under the terms of the Creative Commons Attribution Non-Commercial License (http://creativecommons.org/ licenses/by-nc/3.0/), which permits unrestricted use, distribution, and reproduction in any medium, provided the original work is properly cited. 


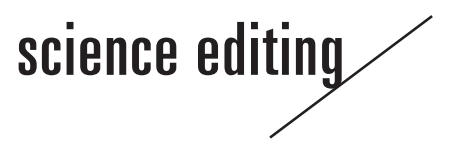

the definition of JATS, its history, and its merits will be presented to help publishers and editors understand the benefits of adopting JATS XML for journal publishing.

\section{Definition of Journal Article Tag Suite}

JATS is an application of NISO Z39.96-2012, which defines a set of XML elements and [1]. In this section, some terms are defined: NISO Z39.96-2012, XML, elements, attributes, tagging, and three article models.

National Information Standards Organization Z39.98-2012 Initially, NISO was where content publishers, libraries, and software developers resorted to for IT industry standards that would allow them to work together [2]. NISO is an US-based organization, whereas ISO is the International Standard Organization. Occasionally, NISO applications are adopted by ISO. In 1935, the first standard by NISO was published as Z39.1, entitled "Reference data for periodicals." The number after "Z39" is changed when new standards are published. For example, NISO Z39.98-2012 is an "Authoring and interchange framework for adaptive XML publishing specification." The last four digits, "2012" in this case, denote the year of the publication.

\section{Extensible markup language}

$\mathrm{XML}$ is a markup language that defines a set of rules for encoding documents in a format that can be read by humans as well as machines. It describes not only the presentation format of content, but also its attributes. Every unicode language is supported and therefore, all character-based languages in the world can be presented via XML on a web site. XML was developed by the XML Working Group under the auspices of the W3C (World Wide Web Consortium) in 1996 [3].

\section{Element}

An element is a logical document component that begins with a start tag and terminates with a matching end tag. For example, consider the following elements:

$<$ country $>$ Korea $</$ country $>$

$<$ email $>$ shuh@hallym.ac.kr </email $>$

The tags < country $>$ and $<$ email $>$ are start tags; the tags $</$ country $>$ and </email $>$ are end tags; and "Korea" and "shuh@hallym.ac.kr" are contents. In certain cases, empty element tags are used, for example, consider the following tags.

$<$ fig $>$

$<$ graphic xlink:href="sun.jpg" $/><$ label $>$ Photo of Sun Huh $</$ label $>$

$</$ fig $>$

\section{Attribute}

An attribute is a markup structure that comprises a name and value pair in the element.

For example, in the element <graphic xlink:href="sun. jpg"/>, xlink:href= "sun.jpg" is the attribute.

The attribute's name is $x l i n k: h r e f$ and its value is sun.jpg.

\section{Tagging}

Tagging is the process of adding tags. A tag is a markup construct that begins with " $<$ " and ends with " $>$." There are three types of tags: start, end, and empty. An empty tag exists as a single tag, i.e., there is neither a start tag nor an end tag. Examples of the three tags are given below.

Start tags: < country $>$, < email $>$

End tags: $</$ country $>$, </email $>$

Empty tags: < graphic xlink:href= "sun.jpg"/ >

Three articles models

The first model is the Journal Archiving and Interchange Tag. Set for the preservation of the intellectual content of journals. The second model is the Journal Publishing Tag Set, which is a moderately prescriptive set optimized for archives such as PubMed Central or ScienceCentral. The third model is the Article Authoring Tag Set that was optimized for authorship of new journal articles. From these three models, the second model is widely used by journals because the deposition to archives is either necessary or mandatory.

\section{History of Journal Article Tag Suite Extensible Markup Language Development}

JATS was developed by the NISO Working Group and published as NISO Z39.96-2012 on August 19, 2012. In 2000, the United States National Center for Biological Information (NCBI) launched PubMed Central (PMC) with two journals: Proceedings of the National Academy of Sciences (PNAS) and Molecular Biology of the Cell [4]. For the presentation of PMC XML files, a tag suite was developed. The first one is pmc-1. dtd. In 2003, NCBI released the National Library of Medicine (NLM) Archiving and Interchange Tag Suite or NLM data type definition (DTD) version 1.0 including the first and second article models. In 2005, the third article model was included and released as version 2.1. In November 2008, version 3.0 was released. After the additional comment of version 3.0, JATS XML was released, which corresponds to NLM DTD 3.1. The most remarkable characteristic of JATS version 1.0 is an acceptance of all character languages in the world, which was accomplished by adding language encoding [5]; therefore, it was possible to establish ScienceCentral as a free, open-access, full-text archive of scientific society journal liter- 
ature at the Korean Federation of Science and Technology Societies regardless of the journals' languages available at http:// e-sciencecentral.org.

ISO Standards Tag Set (ISOSTS) was developed in 2011 by the ISO Central Secretariat. ISOSTS is based on NISO Z39.96 JATS: Journal Article Tag Suite [6]. However, there are some modifications in ISOSTS. New elements are introduced, for example, $<$ annex-type $>$ or $<$ iso-meta $>$. Further, JATS elements are also modified. For example, $<$ ref $>$ is used to exclude notes from the citation content.

\section{Merits of Producing Journal Article Tag Suite Extensible Markup Language}

It is not common to find a JATS XML-based homepage for society journals, except in Korea. In Korea, most medical journals have adopted the JATS XML-based homepage. In other scientific fields, only a small portion of society journals have adopted it. For editors to adopt JATS XML for their journals, they must be convinced of its merits. The following can be suggested as evidence:

First, if JATS XML files are produced for each article, then the journal can be included in international full-text databases such as PMC or ScienceCentral. When a journal is included in those databases, it can be crawled immediately by Google Scholar so that there is a greater chance for each article to be exposed to researchers globally. The more the frequency of exposure increases, the more the impact factor elevates [7]. The quality of journal articles is gauged by the impact factor or citation frequency, and therefore, the exposure platform is important. In particular, ScienceCentral accepts articles related to all scientific fields regardless of language. It also provides a translation function in cooperation with Google; therefore, local-language journals can be circulated to the global scientific arena.

Second, authors who have published research results supported by the United States National Institute of Health need to deposit their articles to PMC. The publisher should be able to produce JATS XML files to deposit these articles although the journal is not open access because it is necessary to fit to the author's request.

Third, the journal's homepage can be promoted on an international level, such as those of international commercial printing companies. The closing of print journals and the increase in online-only publishing is a recent trend in the scholarly journal market. The user-friendliness of the journal homepage is of critical importance. The success of open access online-only journals has accelerated this trend. The traditional format of publishing a journal in print and online has rapidly moved to an online-only format. The online-only format also satisfies author requirements such as rapid publication after submission.

Fourth, it is considerably easy to convert files to another compatible viewer formats such as PubReader (Fig. 1) and

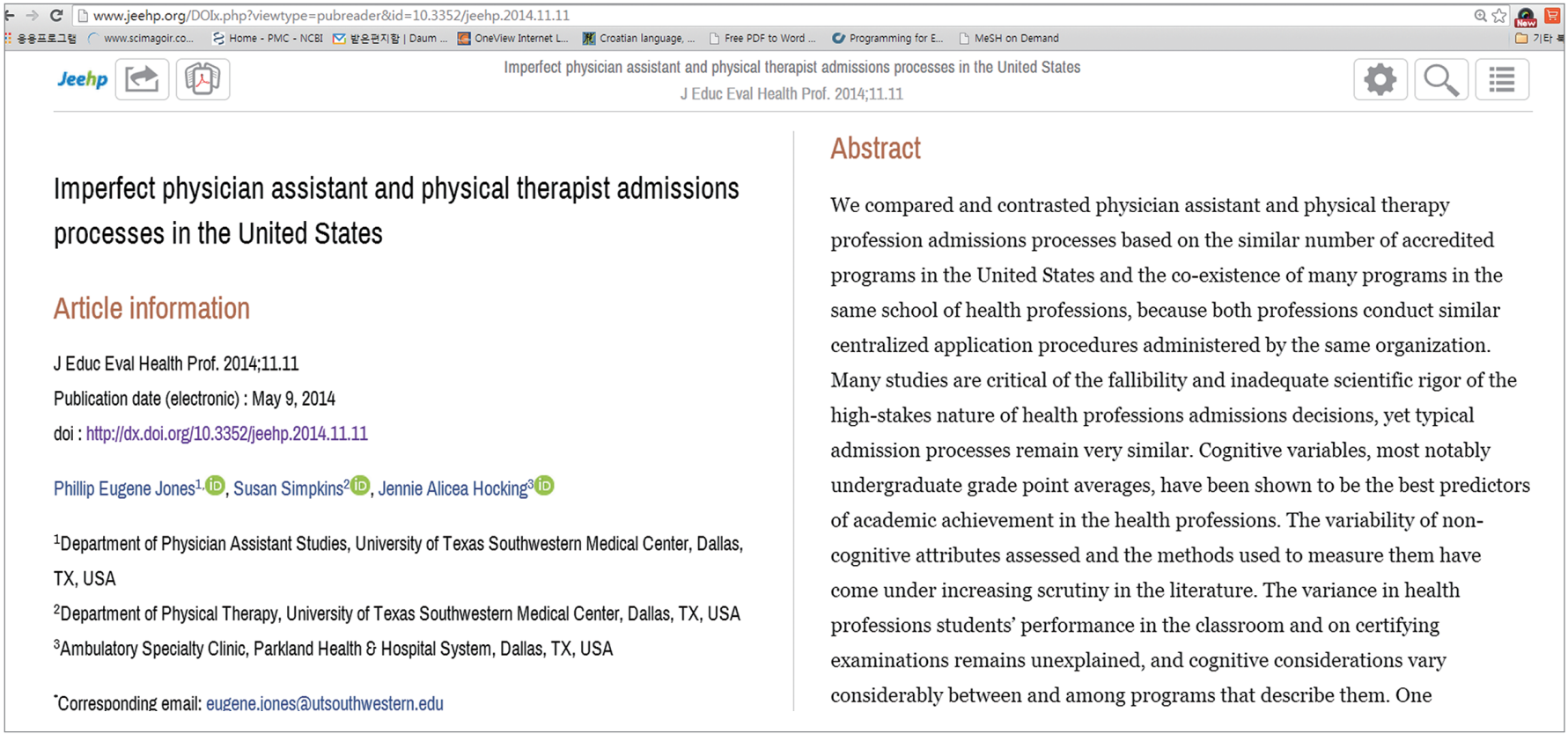

Fig. 1. PubReader format of the Journal Article Tag Suite extensible markup language-based article (Available from: J Educ Eval Health Prof 2014;11: 11. http:// dx.doi.org/10.3352/jeehp.2014.11.11). 


\begin{tabular}{|c|c|c|}
\hline (5) chrome://epubreader/content//reader.xul?id=2 & & $\hat{\omega} \nabla c$ \\
\hline $\begin{array}{l}\text { Article information } \\
\text { INTRODUCTION } \\
\text { CURRENT ADMISSIONS PROCEDURES } \\
\text { PREDICTORS OF ACADEMIC SUCCESS } \\
\text { VALIDITY OF NON-COGNITIVE VARIABLES } \\
\text { DESIRED NON-COGNITIVE VARIABLES IN PA AND } \\
\text { PT ADMISSIONS } \\
\text { LIMITATION OF COGNITIVE AND NON-COGNITIVE } \\
\text { VARIABLES WITH REGARD TO PREDICTION } \\
\text { CONCLUSION } \\
\text { Footnotes } \\
\text { SUPPLEMENTARY MATERIAL } \\
\text { SUPPLEMENTARY MATERIAL } \\
\text { ReferenceS }\end{array}$ & 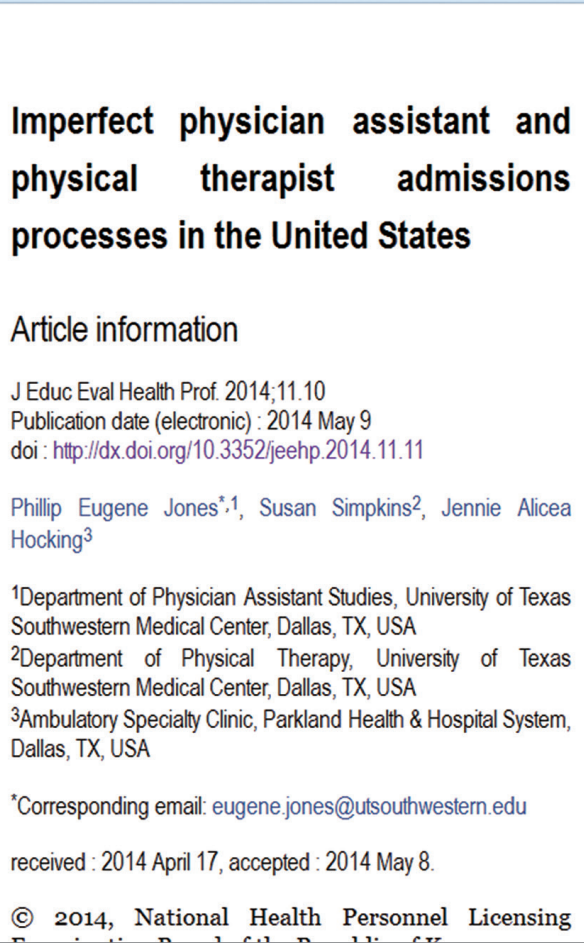 & $\begin{array}{l}\text { We compared and contrasted physician assistant } \\
\text { and physical therapy profession admissions } \\
\text { processes based on the similar number of } \\
\text { accredited programs in the United States and the } \\
\text { co-existence of many programs in the same school } \\
\text { of health professions, because both professions } \\
\text { conduct similar centralized application procedures } \\
\text { administered by the same organization. Many } \\
\text { studies are critical of the fallibility and inadequate } \\
\text { scientific rigor of the high-stakes nature of health } \\
\text { professions admissions decisions, yet typical } \\
\text { admission processes remain very similar. Cognitive } \\
\text { variables, most notably undergraduate grade point } \\
\text { averages, have been shown to be the best predictors } \\
\text { of academic achievement in the health professions. } \\
\text { The variability of non-cognitive attributes assessed } \\
\text { and the methods used to measure them have come } \\
\text { under increasing scrutiny in the literature. The } \\
\text { variance in health professions students' } \\
\text { performance in the classroom and on certifying } \\
\text { examinations remains unexplained, and cognitive } \\
\text { considerations vary considerably between and } \\
\text { among programs that describe them. One } \\
\text { uncertainty resulting from this review is whether or } \\
\text { not desired candidate attributes highly sought after } \\
\text { by individual programs are more student-centered } \\
\text { or graduate-centered. Based on the findings from } \\
\text { the literature, we suggest that student success in } \\
\text { the classroom versus the clinic is based on a } \\
\text { a. }\end{array}$ \\
\hline
\end{tabular}

Fig. 2. ePub 3.0 format of the Journal Article Tag Suite extensible markup language-based article (Available from: J Educ Eval Health Prof 2014;11: 11. http:// dx.doi.org/10.3352/jeehp.2014.11.11).

epub 3.0 (Fig. 2). These new formats are convenient because the size of the font does not change across various reader platforms such as laptops, smart pads, smart phones, smart televisions, and other viewer tools.

Fifth, JATS XML conversion to another XML format can be performed automatically through a filter program; for example, to digital object identifier (DOI) CrossRef XML, CrossMark XML, or FundRef XML. These three services are provided by CrossRef [8]. Since new technological standards have been suggested and implemented immediately by scholarly journals, editors should adopt JATS XML to easily introduce new standards to their journals.

Sixth, it is possible to realize the cited-by function and reference hyperlink via DOI by adding an application programming interface.

Seventh, an error in the article text can be detected and revised by producing JATS XML because an error in the XML context can be easily determined, even though it is difficult in the usual manuscript editing process. For example, in the year element, if 2024, 2018, or 204 were input, it can be automatically checked by a program.

There may be other merits of JATS XML production. Cur- rently, PDF files can be generated from JATS XML files through a filtering program. The upgraded JATS versions may provide functions that are currently unimaginable.

\section{How Should Society Journal Editors Plan to Adopt Journal Article Tag Suite Extensible Markup Language?}

If editors decide to adopt JATS XML, what shall they do? First, they should persuade their publisher to accept JATS XML. Of course, some editors may not acknowledge the importance of JATS XML production, in which case there is no need for them to act. This problem may be resolved by the next editor who better understands the trends of the journal market.

Second, when the publisher agrees to invest in JATS XML production, the editor must choose someone to implement JATS XML. If it is a large society that publishes more than five journals or the number of articles per year is more than 1,000, then it would be feasible for the society to employ a specialist to produce XML files after installing the XML production program; however, the above case is rare except for a very few societies. The easy and effective method is to enter in a con- 
tract with commercial companies or non-profit institutes to produce JATS XML files. In Japan, Japan Science and Technology Agency has produced JATS XML and deposited the files to J-STAGE since 2012 [9]. Commercial companies that produce JATS XML files can be searched easily on the web.

Third, the editor should decide with whom he or she works. If a company has succeeded in depositing XML files to ScienceCentral or PMC, the company is considered reliable. Apart from PMC XML, the ability to produce table XTHML, MathML, and ChemML should be considered because, in 2013, PMC started accepting XML files that include table XTHML, MathML, and ChemML. Furthermore, similar to PMC, ScienceCentral accepts only complete JATS XML files.

Fourth, the cost of XML production should be negotiated. The cost varies according to the company. The internationally accepted price is 50 to 60 US dollars per article. It depends on the publisher's budget and the company's technological excellence. Some companies provide additional functions of JATS XML without an extra cost, whereas others produce only XML files.

Fifth, after the production of JATS XML files, editors should dispatch a request for an inclusion in the JATS XML-based full-text databases. In addition, the journal homepage should be re-constructed. Other functions should be developed by the society itself or by a commercial company. Editors should listen to the feedback from their society's members and make appropriate revision when necessary.

In Korea, the most common hindrance to adopting JATS $\mathrm{XML}$ is a lack of knowledge about JATS XML on the part of editors. If an editor wants to produce a JATS XML file, it is easy and simple because the cost of JATS XML production is considerably reasonable. The price varies according to the number of tables, chemical formulae, and mathematical formulae in the article. Furthermore, because the Korean government supports the publication of society journals, budget is not a problem for editors in Korea.

\section{Conclusion}

To compete with other scholarly journals throughout the world, a society journal has certain handicaps: a shortage of professional editors, managing editors, and IT engineers. Therefore, the society journal editors should be more knowledgeable than those of commercial printing company on a variety of topics in the field of journal publishing. Among them, JATS is one of the most important concepts for journals to survive in the internet age, where most articles are connected to each other. Editors should not hesitate and act immediately to adopt the JATS XML technology. The adoption of this technology will improve the satisfaction level of au- thors, readers, and society members who love the journal.

\section{Conflict of Interest}

No potential conflict of interest relevant to this article was reported.

\section{Acknowledgments}

This work is supported by a research grant of the National Research Foundation of Korea (policy research-2013-003-academic infrastructure promotion) and the Korean Federation of Science and Technology Societies (KOFST-2013), government of the Republic of Korea.

\section{References}

1. National Center for Biotechnology Information, United States National Library of Medicine. Journal Article Tag Suite [Internet]. Bethesda: National Center for Biotechnology Information, United States National Library of Medicine; 2013 [cited 2014 Jun 1]. Available from: http://jats. nlm.nih.gov/

2. National Information Standards Organization. Welcome to NISO [Internet]. Baltimore: National Information Standards Organization; 2014 [cited 2014 Jun 1]. Available from: http://www.niso.org/

3. Wikipedia. XML [Internet]. [place unknown]: Wikipedia; 2014 [cited 2014 Jun 1]. Available from: http://en.wikipe dia.org/wiki/XML/

4. Beck J. NISO Z39.96 the Journal Article Tag Suite (JATS): what happened to the NLM DTDs? J Electron Publ 2011; 14:106. http://dx.doi.org/10.3998/3336451.0014.106

5. Huh S, Choi TJ, Kim SH. Using Journal Article Tag Suite extensible markup language for scholarly journal articles written in Korean. Sci Ed 2014;1:19-23. http://dx.doi.org/ 10.6087/kcse.2014.1.19

6. International Organization for Standardization. ISO Standards Tag Set (ISOSTS) version 1.0: a customization of NISO JATS version 0.4 [Internet]. Geneva: International Organization for Standardization; 2012 [cited 2014 Jun 1]. Available from: http://www.iso.org/schema/isosts/v1.0/ doc/index.html

7. Jeong GH, Huh S. Increase in frequency of citation by SCIE journals of non-Medline journals after listing in an open access full-text database. Sci Ed 2014;1:24-6. http:// dx.doi.org/10.6087/kcse.2014.1.24

8. Lammey R. CrossRef developments and initiatives: an update on services for the scholarly publishing community from CrossRef. Sci Ed 2014;1:13-8. http://dx.doi.org/10. 
6087/kcse.2014.1.13

9. Katoh K, Kobayashi T, Kitazawa M. Reducing costs and expanding XML submissions with PDF to JATS conversion. Journal Article Tag Suite Conference (JATS-Con)
Proceedings 2012 [Internet]. Bethesda: National Center for Biotechnology Information; 2012 [cited 2014 Jun 1]. Available from: http://www.ncbi.nlm.nih.gov/books/NBK 100490/ 